Z Herz- Thorax- Gefäßchir 2019 · 33:147-148 https://doi.org/10.1007/s00398-019-0297-3 Online publiziert: 27. Februar 2019

(c) Springer Medizin Verlag $\mathrm{GmbH}$, ein Teil von Springer Nature 2019

Maude E. Abbotts Weg, eine Pionierin im Bereich der Kardiologie zu werden, war nicht einfach. Sie wurde am 18.03.1869 in St. Andrews East, Quebec, Kanada, ungefähr 40 Meilen nordwestlich von Montreal als Maude Elizabeth Seymour Babin geboren, zwei Jahre nach ihrer Schwester Alice. Der Vater verließ nach tragischen Vorkommnissen die Familie noch vor Maudes Geburt. Die Mutter starb an einer Tuberkulose, als Alice gerade 2 Jahre und Maude eben 7 Monate alt war. Im Alter von 62 Jahren adoptierte die Großmutter mütterlicherseits, Frances Abbott, beide Mädchen, die ihre eigenen 9 Kinder zuvor verloren hatte. Die Namensänderung von Babin auf Abbott erstritt sie durch einen speziellen Beschluss des kanadischen Parlaments.

Maudes schulische und anschließende akademische Ausbildung erfolgte in Montreal, wo sie nach ihrer Graduierung als Bachelor of Arts (BA) an der McGill University als Beste ihres Jahrgangs auch die Abschlussrede hielt. 1894 schloss sie als einzige Frau den Studiengang Medizin ebenfalls als Beste mit einem Medical Doctor (MD) und etlichen Preisen ab. Da sie als Frau jedoch keine Stelle an der McGill University für eine PostgraduateAusbildung bekommen konnte, trat sie, in Begleitung ihrer Schwester Alice, unmittelbar danach eine zweijährige Reise durch die großen europäischen Universitätsstädte London, Zürich, Heidelberg und Wien an. Alice, die als angehende Pianistin unterwegs war, erkrankte in Wien an einer Diphtherie, die ihre bereits zuvor bestehende psychische Erkrankung auf Dauer erheblich komplizierte.

Im Frühjahr 1897, zurück in Montreal, eröffnete Maude zunächst eine private Praxis für Frauen und Kinder, begann jedoch bald, zusätzlich und auf freiwil-

\author{
H. E. Ulmer \\ Heidelberg, Deutschland
}

\title{
Maude E. Abbott
}

liger Basis, eine wissenschaftliche Tätigkeit am dortigen Royal Victoria Hospital. Der erste von ihr verfasste Vortrag über „Sogenannte accidentelle Herzgeräusche" musste jedoch in der Medico-Surgical Society von ihrem Freund, dem späteren Dekan der McGill Universität, Charles Martin, gehalten werden, da Frauen nicht zugelassen waren. Allerdings wurde sie anschließend von den beeindruckten Mitgliedern der Society als erste Frau gebeten, Mitglied ihrer Gesellschaft zu werden. Selbst jetzt erhielt sie von der McGill Universität keine Stelle als Assistentin, sondern lediglich das Angebot einer eher symbolischen Position als „assistant curator“ des „Medizinischen Museums“, einer Sammlung seltener anatomischer Präparate, und wurde 1901 auch zu dessen „chief curator“ ernannt.

In den folgenden Jahren ordnete und katalogisierte Maude Abbott die seit

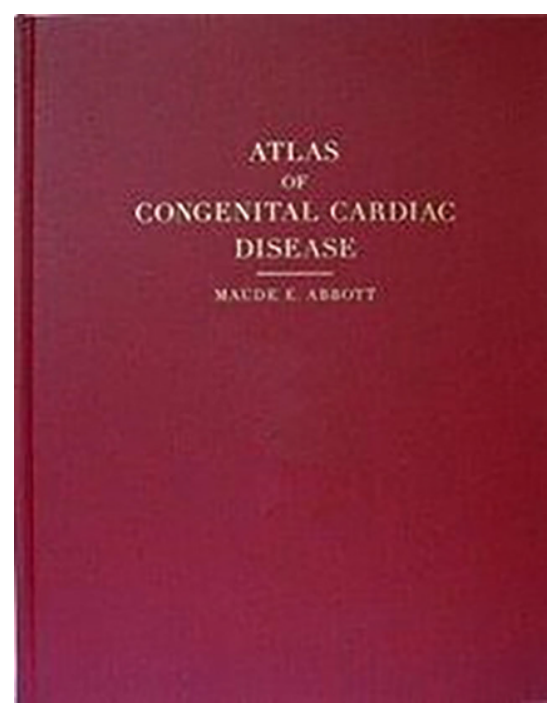

Abb. $1 \Delta$ Cover des Atlas über Anatomie und Pathologie angeborener Herzfehler von Maude Abbott, veröffentlicht 1936 von der American Heart Association mehr als 60 Jahren gesammelten anatomischen Präparate des Museums. Diese bestanden zum großen Teil aus Herzen, die noch von dem späteren Internisten Sir William Osler konserviert worden waren. Mit der Beschreibung eines als infektiöse Myokarditis falsch deklarierten Herzens, das eine der komplexesten Fehlbildungen überhaupt aufwies, dem „Holmes heart“, war Maude Abbotts Interesse an einer Systematik angeborener Herzfehler endgültig geweckt. Sehr bald wurde sie von William Osler gebeten, in dem von ihm geplanten und herausgegebenen siebenbändigen internistischen Standardwerk den Beitrag über angeborene Herzfehler zu übernehmen. Der Umfang betrug in der 1. Ausgabe des Jahres 1908 noch 22 Seiten, in der 3. Ausgabe des Jahres 1927 jedoch bereits mehr als 200 Seiten. Ihre weiteren wissenschaftlichen Arbeiten machten sie bald zur unumstrittenen Autorität auf

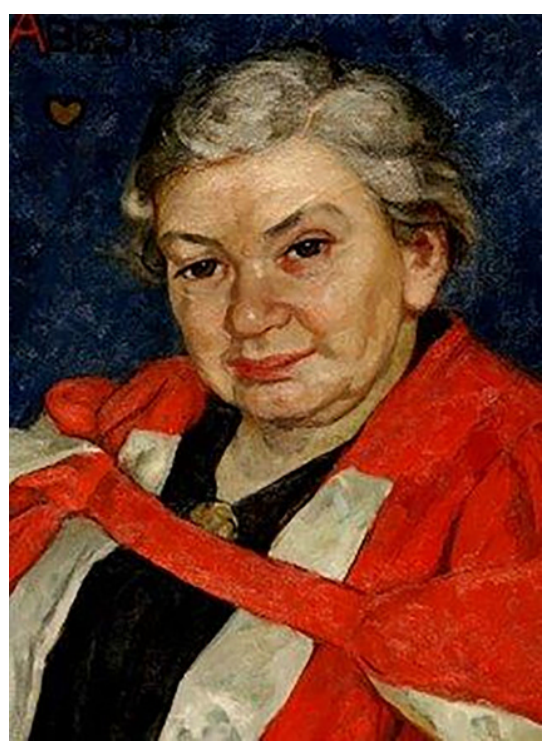

Abb. 2 ^ Maude E. Abbott (1869-1940) 


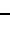

diesem Gebiet in den frühen Jahren des diesem Gebiet in den frühen Jahren des 20. Jh.

1910, d.h. 8 Jahre, bevor die erste Frau zur Weiterbildung in der Medizin an der McGill University zugelassen wurde, wurde sie, zwar erneut symbolisch, zum „Ehren-Lektor“ ernannt, erhielt jedoch wieder keine offizielle akademische Position. Erst nachdem sie 1923 zwischenzeitlich einen Ruf auf die Professur für Pathologie des Woman's Medical College of Pennsylvania, USA, angenommen hatte, konnte sie zwei Jahre später, allerdings auf die rangniedrigere Position eines ,,assistant professor", nach Montreal zurückkehren.

Bereits zuvor war es Maude Abbotts größter Wunsch geworden, ein zusammenfassendes Handbuch über pädiatrische Kardiologie zu schreiben. Dieser Wunsch blieb ihr jedoch versagt. Sie erlebte es jedoch, als 1936 der erste Kernteil ihres Werks als „Atlas of Congenital Cardiac Disease“ als interne Monographie von der American Heart Association gedruckt wurde (• Abb. 1). Eigentlich handelte es sich dabei lediglich um einen Katalog zu ihrer Sammlung. Erst die um Jahre später im wissenschaftlichen Buchhandel erschienenen Ausgaben waren ergänzt durch grafische Darstellungen zum besseren Verständnis der Anatomie, um klinische Angaben zum Fall sowie um Fotografien von zugehörigen Röntgenaufnahmen und Elektrokardiogrammen. Die ganze Ausstellung, die mehr als 1000 Präparate umfasste, wurde verpackt, und Maude Abbott reiste damit zu pathologischen Demonstrationen an die großen Universitäten in den Vereinigten Staaten und in Europa. Diese Anstrengung festigte ihren Ruf als wissenschaftliche Autorität auf beiden Seiten des Atlantiks. Publiziert wurde der an sich die Pathologie des Herzens thematisierende Atlas in der ersten Auflage 1936 von der American Heart Association, später noch mehrfach, zuletzt 2006.

1934 verstarb ihre Schwester Alice, deren Fürsorge und Pflege Maude während ihres gesamten gemeinsamen Lebens übernommen hatte, zu Hause in ihren Armen.

1936 war ein entscheidendes Jahr für Maude Abbott. Im Alter von 67 Jahren wurde ihr der Eintritt in den Ruhe- stand nahegelegt, der dann auch, wenngleich gegen ihren eigenen Willen, erfolgte. Trotz ihrer Verdienste und ihrer weltweiten Reputation verweigerte die McGill Universität ihr als Frau die Anerkennung einer "full professorship“bis zum Schluss. $\mathrm{Zu}$ ihrer Entlassung wurde ihr jedoch die höchste Auszeichnung der Universität, die Verleihung eines Ehrendoktors der (beachte) juristischen Fakultät, zuteil. Ausführliche Reisen führten sie anschließend durch große Teile Kanadas und der USA, wo sie zahlreiche Ehrungen erfuhr.

Im Sommer 1940 erlitt sie einen Schlaganfall, von dem sie sich nicht recht erholte. Am 2. September 1940 verstarb Maude Abbott im Alter von 71 Jahren zuhause in Montreal an den Folgen einer Gehirnblutung, die sie bereits im Juli des Jahres erlitten hatte, sich davon aber nie mehr erholte.

In Mexico City hängt im National Institute of Cardiology das bekannte Fresko des Malers Diego Rivera, auf dem die wichtigsten Mediziner und Ärzte aller Zeiten bis zur Mitte des 20. Jh. dargestellt sind. Maude Abbott ist darauf als einzige Frau und einzige kanadische Persönlichkeit abgebildet (• Abb. 2). 1994 wurde sie, mehr als 50 Jahre nach ihrem Tod, in die Canadian Medical Hall of Fame aufgenommen.

\section{Korrespondenzadresse}

\section{Prof. Dr. H. E. Ulmer}

Am Aukopf 5, 69118 Heidelberg, Deutschland herbert_ulmer@t-online.de

\section{Autoren WERKSTATT \\ GRATIS \\ Ein Service für Autoren von Springer Medizin

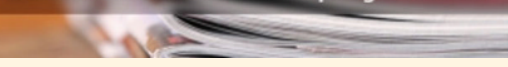 \\ Fortbildungen für Autoren und Gutachter}

Die ersten Veröffentlichungen sind für jeden Mediziner ein wichtiger Schritt in seiner Karriere als Wissenschaftler. Wissenschaftliche Artikel sind entscheidend dafür, dass die eigene Arbeit in der Community wahrgenommen wird. Es geht darum, die eigenen Ideen national und international auszutauschen und sicherzustellen, dass die Ergebnisse Wirkung erzielen. Die Online-Kurse der Autorenwerkstatt helfen, sich leicht einen Überblick über das Schreiben, Einreichen, Begutachten und Veröffentlichen eines Manuskripts zu verschaffen.

5 Online-Kurse zu den wichtigsten Standards des wissenschaftlichen Publizierens:

- Wie verfasse ich ein Manuskript?

- Writing in English für deutschsprachige Autoren

- Wie funktionieren Publikation und Begutachtung?

- Anleitung zur Open-AccessVeröffentlichung

- Leitfaden zur Peer-ReviewBegutachtung

Für alle, die auf SpringerMedizin.de registriert sind!

Jetzt gratis fortbilden unter www.springermedizin.de/ autorenwerkstatt/ 